\title{
Conditions of appearance of the topological defects of optical indicatrix orientation in the glasses with residual stresses: Movement of the defects under application of external mechanical stress to $\mathrm{CaB}_{4} \mathrm{O}_{7}$ glasses
}

\author{
Vasylkiv Yu., Kryvyy T., Skab I. and Vlokh R. \\ Vlokh Institute of Physical Optics, 23 Dragomanov Street, 79005 Lviv, Ukraine, \\ vlokh@ifo.lviv.ua
}

Received: 23.03 .2016

\begin{abstract}
We have revealed experimentally a movement of the topological defects of optical indicatrix orientation, which takes place in $\mathrm{CaB}_{4} \mathrm{O}_{7}$ glass with residual mechanical stresses under application of external compressive mechanical stresses. The effect is explained basing on the equation of optical indicatrix perturbed by the combination of both residual and external mechanical stresses. The conditions for the appearance of the topological defects with the strength module $|1 / 2|$ in glasses are formulated. Finally, possibilities for the appropriate topological reactions caused by the applied stresses are discussed.
\end{abstract}

Keywords: topological defects, optical indicatrix, residual stresses, glasses

PACS: $78.20 . \mathrm{hb}, 62.40 .+\mathrm{I}, 02.40 . \mathrm{Pc}$

UDC: $535.551+620.171 .5+515.143$

\section{Introduction}

Topological defects (TDs) that appear as a result of some symmetry lowering are known in many branches of physics, including the theory of interaction of forces [1, 2], cosmology [3], and condensed matter physics [4-6]. For example, 2D TDs arising spontaneously in the ferroic materials in the form of domain walls [4]. Probably, the TDs of director orientation appearing in liquid crystals have been studied the best $[5,6]$. Recently we have shown that, under the action of inhomogeneous electric fields or mechanical stresses, TDs of optical indicatrix (OI) orientation can appear either in glasses or in crystalline materials [7-12]. Whenever a conically shaped electric field is applied to the crystals of certain point symmetries, a Pockels effect forms the TDs of OI orientation with the strength equal to $\pm 1 / 2$ and a Kerr effect induces the TDs with the strength $\pm 1[7,8]$. We have also found that superposition of the both electrooptic effects can result in the topological reactions of birth, adding and annihilation of the TDs [9-11].

According to our experimental data [12-15], the mechanical stresses of bending or torsion can also induce the TDs with the half-integer strength in many situations. In particular, the TDs of OI orientation with the strength module $|1 / 2|$ appear in the amorphous glass media with residual stresses [16]. Notice that the general conditions needed for these defects to appear have still not be elucidated in the literature. It is known from Ref. [17] that the strengths of those defects, in particular of the TDs arising in $\mathrm{CaB}_{4} \mathrm{O}_{7}$ glass, have the same (positive) signs. Then a removal of the TDs cannot be accompanied by a reaction of annihilation, i.e. the defects can be eliminated either by leaving a sample due to application of some external mechanical stresses - or by vanishing due to annealing. In the former case a movement of the TDs within the sample has to be observed under application of external mechanical stress, although no experimental confirmation of that phenomenon is still available.

Ukr. J. Phys. Opt. 2016, Volume 17, Issue 2 
In this work we clarify the conditions of appearance of the TDs of OI orientation in the glasses with residual stresses and study the movement of TDs in the glass samples under the application of external compressive stresses.

\section{Experimental methods}

In our experiments we used a borate $\mathrm{CaB}_{4} \mathrm{O}_{7}$ glass with the refractive index equal to $n=1.544$ at the light wavelength $\lambda=632.8 \mathrm{~nm}$ [17]. A glass boule was not annealed so as to retain the residual mechanical stresses that produced nonzero optical birefringence inside a sample. The sample was prepared in the shape of parallelepiped, with the sizes $8.53,4.41$ and $3.87 \mathrm{~mm}$ respectively along $Z, Y$ and $X$ axes. The sample was placed onto a stage that allowed application of compressive stresses along the $Z$ axis (see Fig. 1). A wide parallel circularly polarized laser beam $(\lambda=632.8 \mathrm{~nm})$ propagated through the polarization system along the $X$ or $Y$ axes of the sample. The sample was placed in between circular or linear polarizers. The optical output was detected with the aid of a CCD camera linked with a computer.

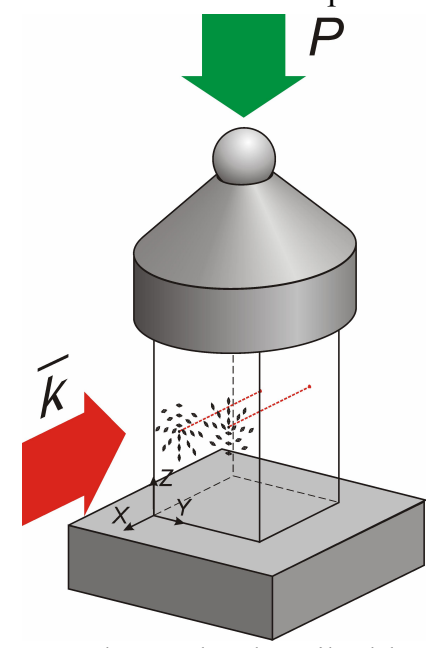

Fig. 1. Schematic view of a sample containing a TD of Ol orientation, which is placed into a setup for applying external pressures: a dashed line corresponds to a chain of defects and ellipses denote the cross-section OI.

The sample can be described by a linear phase retarder model for which the dependence of the output light intensity $I$ on the analyzer azimuth $\alpha$ is expressed as

$$
I=\frac{I_{0}}{2}\{1+\sin \Delta \Gamma \sin [2(\alpha-\zeta)]\}=C_{1}+C_{2} \sin \left[2\left(\alpha-C_{3}\right)\right]
$$

where $\zeta$ is the orientation angle of the OI, $\Delta \Gamma=2 \pi \Delta n d / \lambda$ the optical phase difference, $d$ the sample thickness along the light propagation direction, $\Delta n$ the optical birefringence, $\alpha$ the angle of analyzer orientation, and $I_{0}$ the incident light intensity. After recording and filtering a relevant optical image, the azimuthal dependences of the intensity $I$ are fitted with a sine-squared function for every pixel, with the fitting coefficients

$$
C_{1}=\frac{I_{0}}{2}, C_{2}=\frac{I_{0}}{2} \sin \Delta \Gamma, C_{3}=\zeta .
$$

Then the optical phase difference $\Delta \Gamma$ is given by the coefficients $C_{1}$ and $C_{2}$,

$$
\sin \Delta \Gamma=C_{2} / C_{1}
$$

while the angular orientation of the intensity minimum is determined by the orientation of the principal axis $\zeta$ of the OI and the coefficient $C_{3}$. 
Hence, fitting of the dependences of the light intensity passed through the analyzer upon the azimuth for each pixel of the sample image enables to construct 2D maps of the optical anisotropy parameters of the sample, the optical phase difference and the OI orientation. The optical setup has been described in detail in a number of our earlier works (see, e.g., Refs. [8, 9, 13, 14, 16, 17]). It is worthwhile that the procedures for measuring the phase difference and the OI rotation angle described above are strictly applicable only to the cases of 2D distributions of the optical anisotropy parameters or, at least, under the conditions that the OI orientation does not depend on the spatial coordinate along the direction of light propagation. In case of the residual stresses in our glass sample, the phase difference is very small and so the birefringence distributed in some manner inside the sample does not exceed $\sim 10^{-5}$. The above facts enable one to neglect inhomogeneities of the optical parameters along the light propagation direction while measuring the OI orientation and the phase difference.

\section{Results and discussion}

Spatial maps of the OI orientation and the phase difference are presented respectively in Fig. 2 and Fig. 3. Two pairs of the TDs $\left(\mathrm{TD}_{1}\right.$ and $\mathrm{TD}_{2}$ on an $Y Z$ face, and $\mathrm{TD}_{3}$ and $\mathrm{TD}_{4}$ on a $Z X$ face of the sample) are clearly seen under the condition of zero external mechanical stresses. All of the defects have the strength equal to $1 / 2$, since the OI rotates by the angle 180 deg around the defect core when the tracing angle changes by $360 \mathrm{deg}$ (see Fig. 4). As seen from Fig's. 2, 3, 5, the defect core is characterized by a zero phase difference and uncertainty of the OI orientation angle. According to our recent experimental studies on the same sample [17], all the defects have the positive strengths. Moreover, the dependence of the phase difference on the coordinate in the vicinity of the defect cores is linear. For the distances from the defect cores less than the $\rho(\rho=0.57 \mathrm{~mm})$, the OI rotation angle does not depend on $\rho$ (see Fig. 5). Furthermore, the full intensity extinction of the light emergent from the sample placed in between the crossed linear polarizers, which is observed in the region where the phase difference depends linearly on the coordinates, means that we indeed deal with a 2D stress distribution in this region. Finally, vanishing of the phase difference at the light propagation through the defect cores should imply that our defects are linear ones. In fact, the defects represent linear chains inside the sample, which are surrounded by the region of 2D distribution of the phase difference and, hence, of the residual stresses.

As seen from Fig. 2, application of the compressive mechanical stresses $-3.22 \times 10^{6}$ and $-6.74 \times 10^{6} \mathrm{~N} / \mathrm{m}^{2}$ leads to a sliding movement of the defects inside the sample. The defects $\mathrm{TD}_{1}$ and $\mathrm{TD}_{2}$ move downwards and upwards, respectively. The changes in the positions of these defects occurring with changing stress are shown in Fig. 6 . We have found that the dependence of the displacement ( $d=\beta \sigma_{3}^{\prime}$ ) of these TDs on the compressive stress is almost linear. The modules of the proportionality coefficient $\beta$ are equal to $(6.64 \pm 1.28) \times 10^{-11}$ and $(4.95 \pm 1.44) \times 10^{-10} \mathrm{~m}^{3} / \mathrm{N}$ respectively for $\mathrm{TD}_{1}$ and $\mathrm{TD}_{2}$, i.e. these coefficients differ in their orders of magnitude. Since the movability parameters of those defects are quite different, they cannot originate from some structural imperfections of the glass. The $\mathrm{TD}_{1}$ and $\mathrm{TD}_{2}$ defects tend to leave the sample with increasing pressure (see Fig. 2). However, these defects cease to be detectable already at the mechanical stress $-13.48 \times 10^{6} \mathrm{~N} / \mathrm{m}^{2}$, since the phase difference induced by the external pressure via a piezooptic effect becomes comparable to the phase difference caused by the residual stresses themselves. As a result, we observe appearance of the birefringence along the direction of light propagation through the defect core (see Fig. 5e, f), perturbation of the OI orientation angle and, consequently, smearing of the defects. When the light propagates along the $Y$ direction, the indu- 
ced phase difference becomes comparable to the residual one at still lower pressures $\sigma_{3}^{\prime}$ $\left(-6.74 \times 10^{6} \mathrm{~N} / \mathrm{m}^{2}-\right.$ see Fig. 3). This is because the initial phase difference is here somewhat smaller than that typical for the case of light propagation along the $X$ axis. However, a further increase in the external pressure up to $-13.48 \times 10^{6} \mathrm{~N} / \mathrm{m}^{2}$ induces new defects, $\mathrm{TD}_{5}$ and $\mathrm{TD}_{6}$ (see Fig. $3 \mathrm{e}, \mathrm{f}$ ).
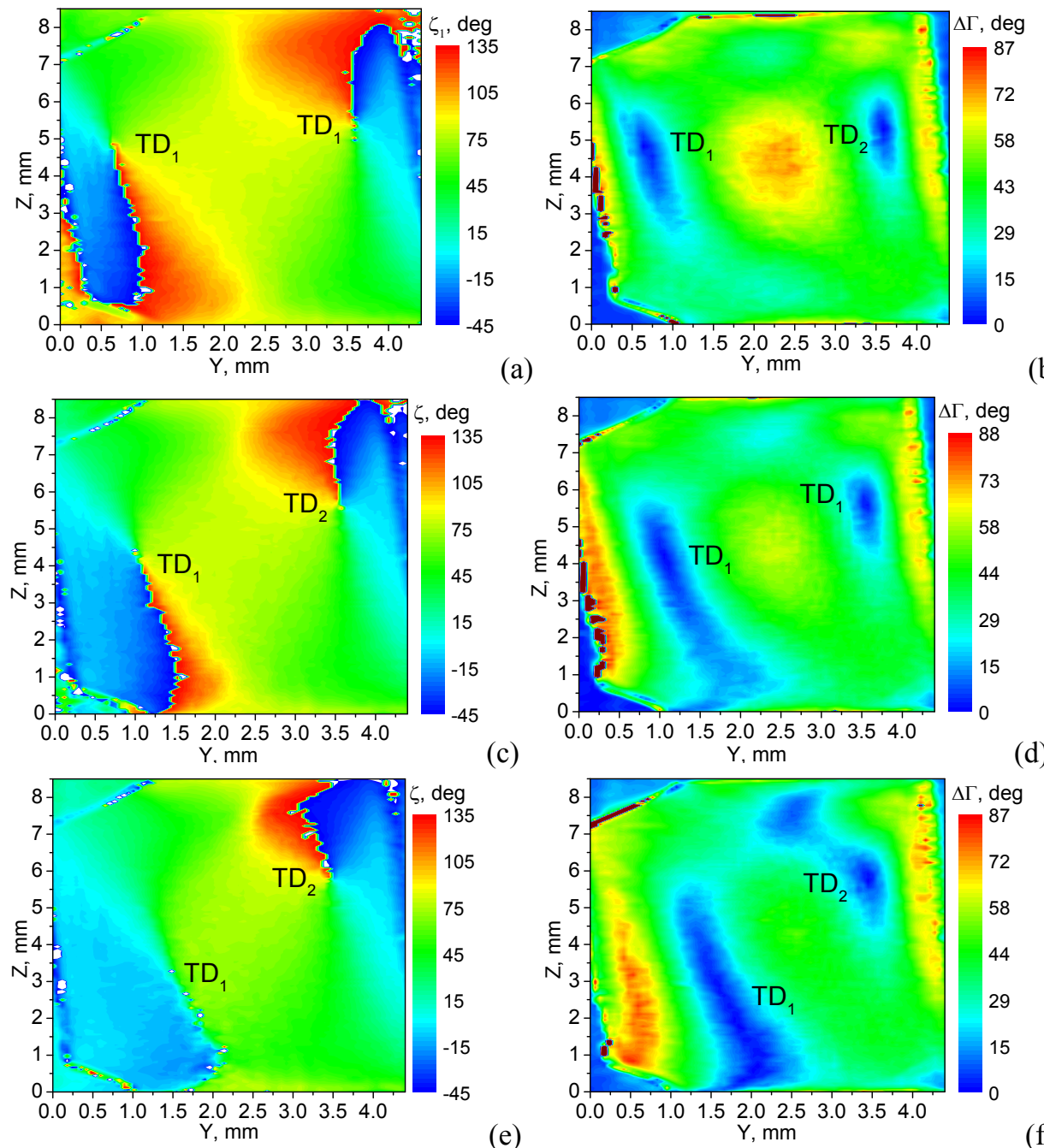

(c)

(b)
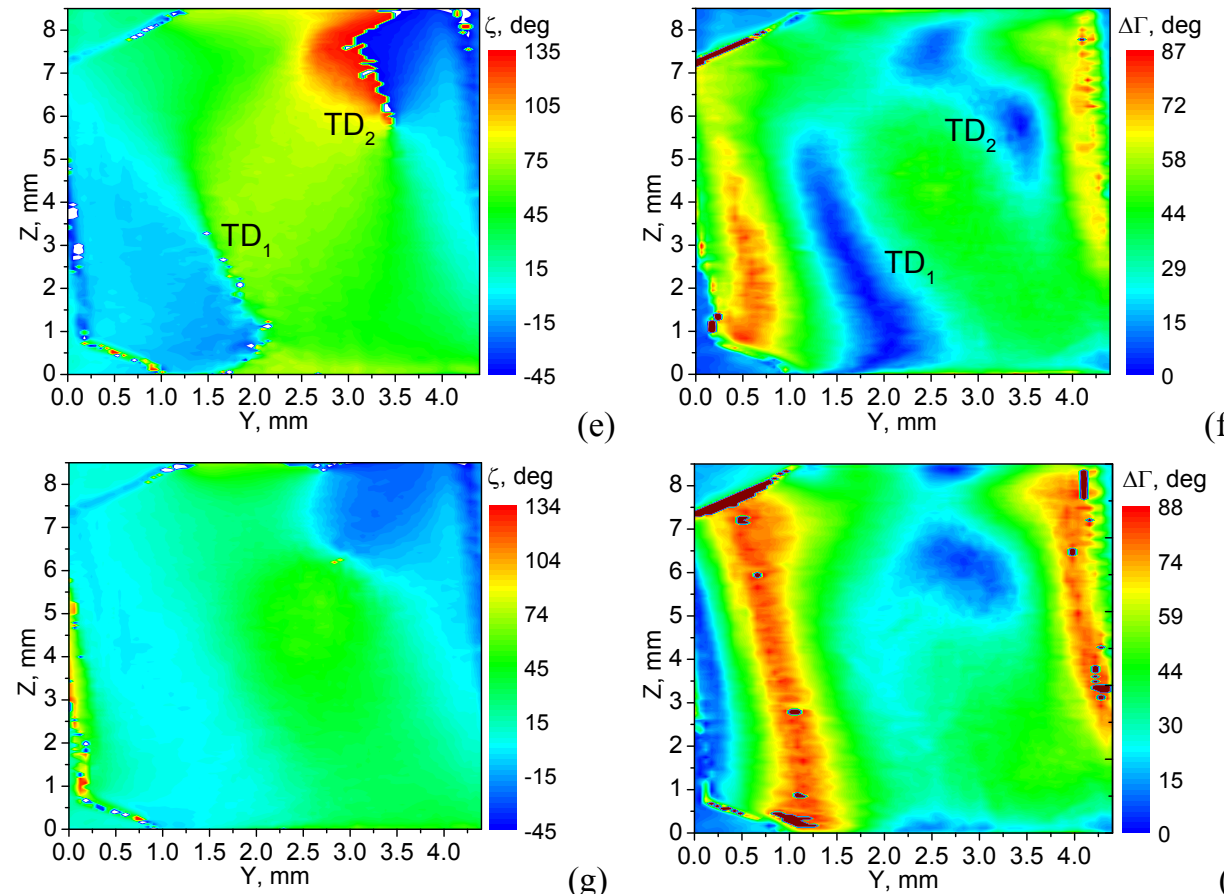

e)

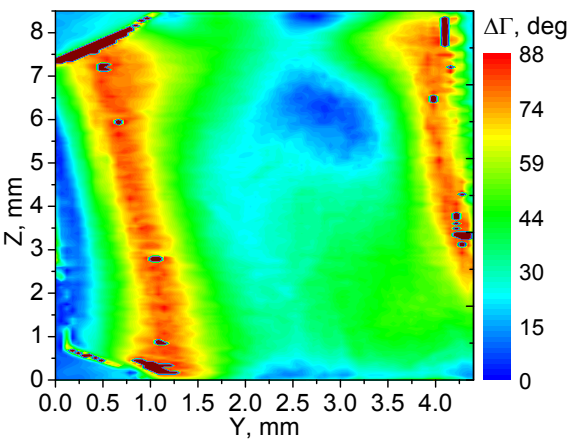

Fig. 2. Spatial maps of the OI orientation angle (a, c, e, g) and the phase difference (b, $d, f, h)$ appearing under application of different mechanical stresses along the $Z$ axis and light propagation along the $X$ axis. The stresses applied to $\mathrm{CaB}_{4} \mathrm{O}_{7}$ glass are equal to $0(\mathrm{a}, \mathrm{b}),-3.22 \times 10^{6} \mathrm{~N} / \mathrm{m}^{2}(\mathrm{c}, \mathrm{d}),-6.74 \times 10^{6} \mathrm{~N} / \mathrm{m}^{2}$ (e, f) and $-13.48 \times 10^{6} \mathrm{~N} / \mathrm{m}^{2}(\mathrm{~g}, \mathrm{~h})$. 

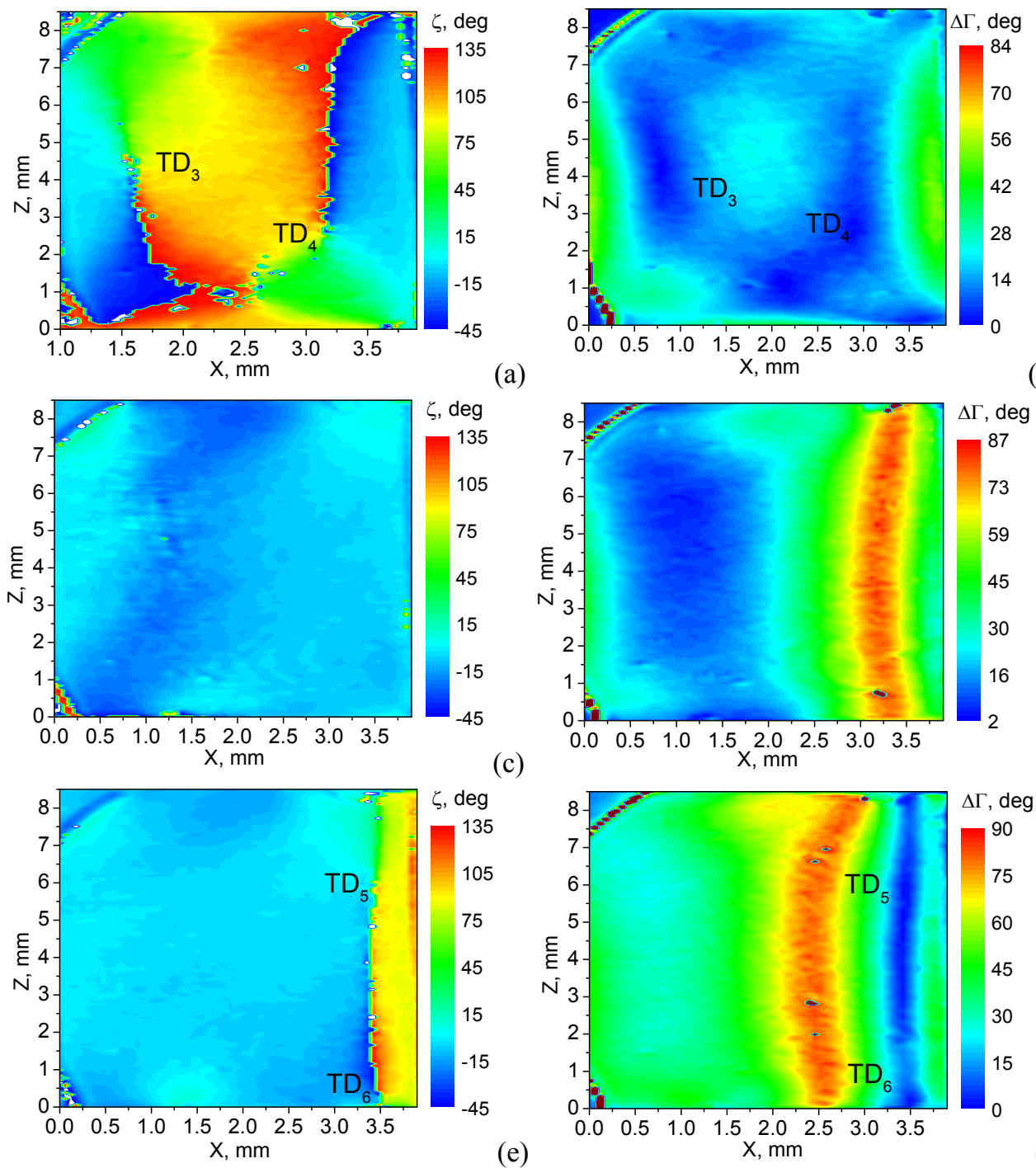

(d)

Fig. 3. Spatial maps of the Ol orientation angle $(a, c, e)$ and the phase difference $(b, d, f)$ appearing under application of different mechanical stresses along the $Z$ axis and light propagation along the $Y$ axis. The stresses applied to $\mathrm{CaB}_{4} \mathrm{O}_{7}$ glass are equal to $0(a, b),-6.74 \times 10^{6} \mathrm{~N} / \mathrm{m}^{2}(\mathrm{c}, \mathrm{d})$ and $-13.48 \times 10^{6} \mathrm{~N} / \mathrm{m}^{2}(\mathrm{e}, \mathrm{f})$.

Let us analyze the reasons for appearance of the TDs of OI orientation in the stressed glasses. The TDs of OI orientation with the strength equal to $\pm 1 / 2$, which are caused by inhomogeneous mechanical stresses, can appear in the glass media if the following equations for the OI orientation angle are satisfied:

$$
\begin{aligned}
\tan 2 \zeta_{1} & =\frac{2 \sigma_{4}(Y, Z)}{\sigma_{2}(Y, Z)-\sigma_{3}(Y, Z)}, \tan 2 \zeta_{2}=\frac{2 \sigma_{5}(X, Z)}{\sigma_{3}(X, Z)-\sigma_{1}(X, Z)}, \text { or } \\
\tan 2 \zeta_{3} & =\frac{2 \sigma_{6}(X, Y)}{\sigma_{1}(X, Y)-\sigma_{2}(X, Y)} .
\end{aligned}
$$

Note that these relations follow from the general equation of OI. Since the coordinate dependences of the phase difference are linear (see Fig. 5), the mechanical stress tensor components (written, e.g., for the $Y Z$ plane and the defect $\mathrm{TD}_{2}$ ) have to depend linearly on the coordinates: 

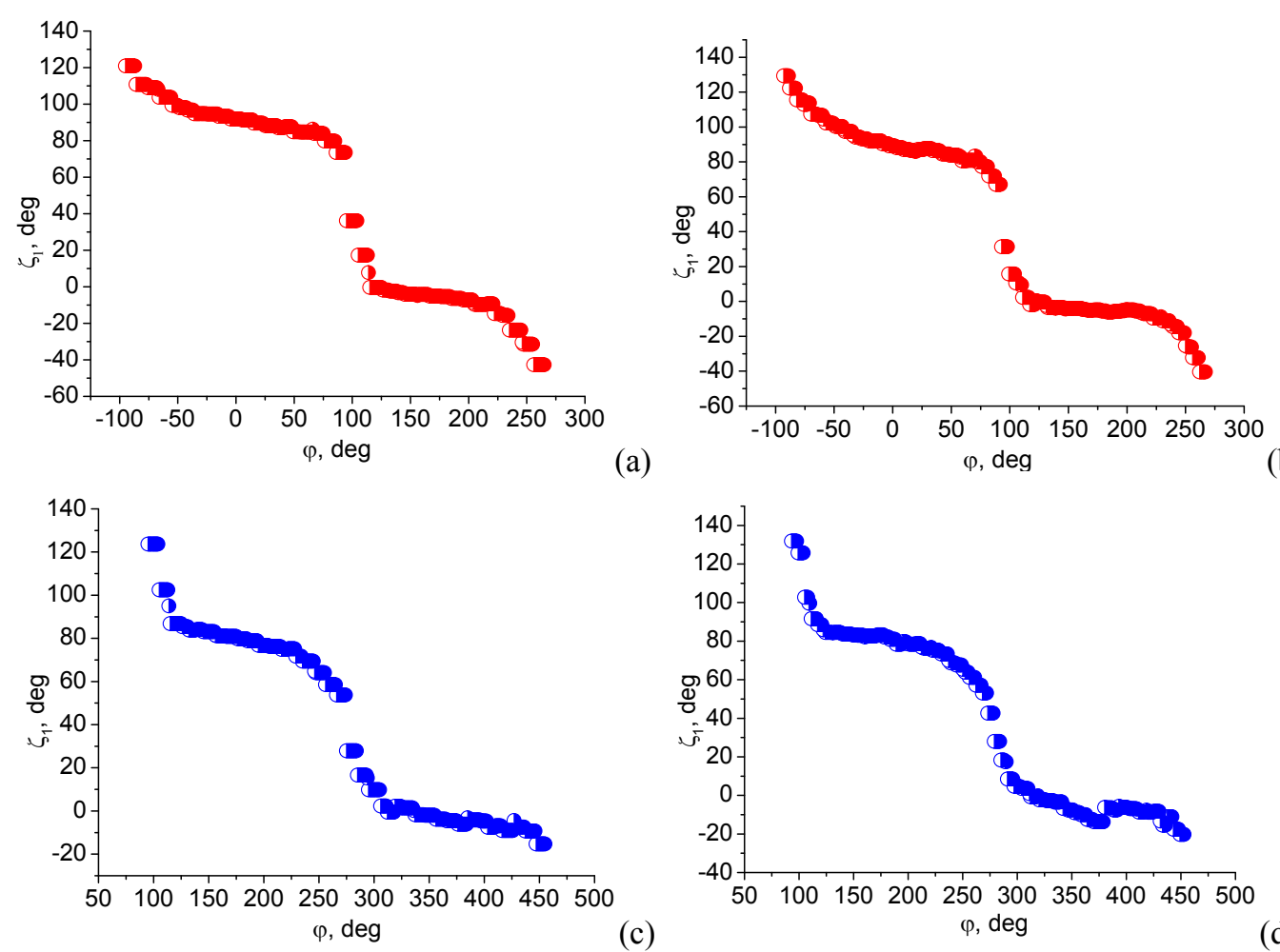

Fig. 4. Dependences of the angle of $\mathrm{Ol}$ rotation around the defect cores in $\mathrm{CaB}_{4} \mathrm{O}_{7}$ glass on the tracing angle for $\mathrm{TD}_{1}(\mathrm{a}, \mathrm{b})$ and $\mathrm{TD}_{2}(\mathrm{c}, \mathrm{d})$, as obtained at the distances $0.34 \mathrm{~mm}(\mathrm{a}, \mathrm{c})$ and $0.57 \mathrm{~mm}(\mathrm{~b}, \mathrm{~d})$ from the defect cores under the condition $\sigma_{3}^{\prime}=0$.

$$
\sigma_{4}=k_{1} Y+k_{2} Z, \sigma_{2}=k_{3} Y+k_{4} Z \text { and } \sigma_{3}=k_{5} Y+k_{6} Z,
$$

where $k_{\mathrm{i}}$ are the proportionality coefficients. Then the equation for the OI cross section may be written as

$$
\left(B_{1}+\pi_{11} \sigma_{2}+\pi_{12} \sigma_{3}\right) Y^{2}+\left(B_{1}+\pi_{12} \sigma_{2}+\pi_{11} \sigma_{3}\right) Z^{2}+2\left(\pi_{11}-\pi_{12}\right) \sigma_{4} Y Z=1,
$$

where $B_{i}$ and $\pi_{i j}$ are components of the optical impermeability tensor and the piezooptic tensor, respectively.

Hence, the birefringence and the angle of OI rotation induced by the residual stresses are given by the relations

$$
\begin{aligned}
& \Delta n_{23}=-\frac{n^{3}}{2}\left(\pi_{11}-\pi_{12}\right) \sqrt{\left.\left(\left(k_{3}-k_{5}\right) Y+\left(k_{4}-k_{6}\right) Z\right)\right)^{2}+4\left(k_{1} Y+k_{2} Z\right)^{2}}, \\
& \tan 2 \zeta_{1}=\frac{2\left(k_{1} Y+k_{2} Z\right)}{\left(k_{3}-k_{5}\right) Y+\left(k_{4}-k_{6}\right) Z} .
\end{aligned}
$$

Notice that, according to Eq. (7), one of the observed properties of TDs, the equality to zero of the birefringence in the TD cores, holds at $Y=Z=0$. After taking the elastostatics equation $\left(\partial \sigma_{k l} / \partial X_{l}=0\right)$ and Eqs. (5) into account, one can find that the equilibrium conditions hold true at $k_{3}=-k_{2}$ and $k_{1}=-k_{6}$. Then we rewrite Eqs. (7) and (8) as

$$
\Delta n_{23}=-\frac{n^{3}}{2}\left(\pi_{11}-\pi_{12}\right) \sqrt{\left(\left(k_{4}+k_{1}\right) Z-\left(k_{2}+k_{5}\right) Y\right)^{2}+4\left(k_{1} Y+k_{2} Z\right)^{2}},
$$




$$
\tan 2 \zeta_{1}=\frac{2\left(k_{1} Y+k_{2} Z\right)}{\left(k_{4}+k_{1}\right) Z-\left(k_{2}+k_{5}\right) Y} .
$$

As found in Ref. [15], the TDs with the strength $\pm 1 / 2$ appear when Eq. (10) acquires the following general form:

$$
\tan 2 \zeta_{1}=\frac{2\left(k_{1} Y \pm k_{2} Z\right)}{\left(k_{4}+k_{1}\right) Z \mp\left(k_{2}+k_{5}\right) Y}
$$
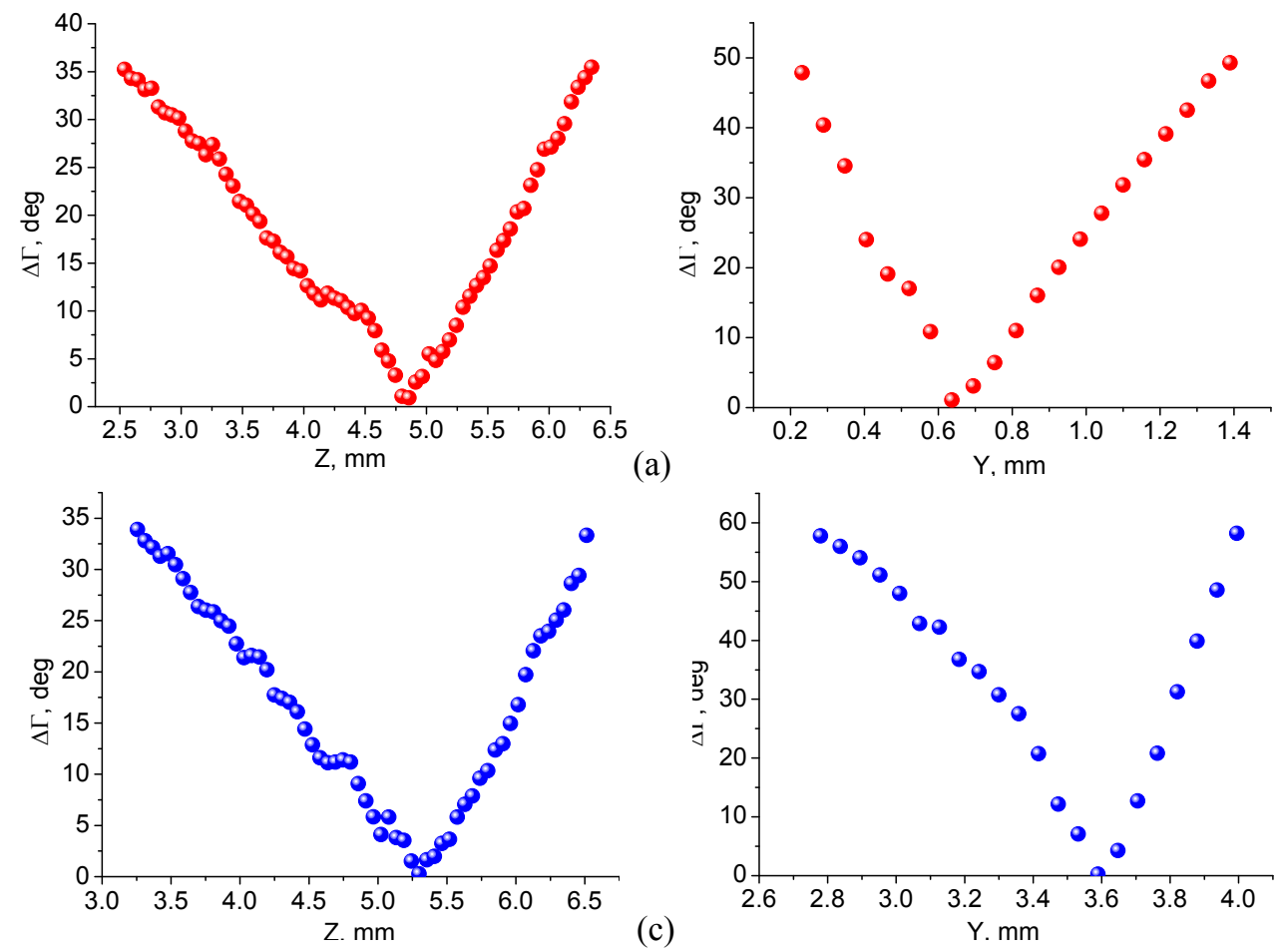

(b)
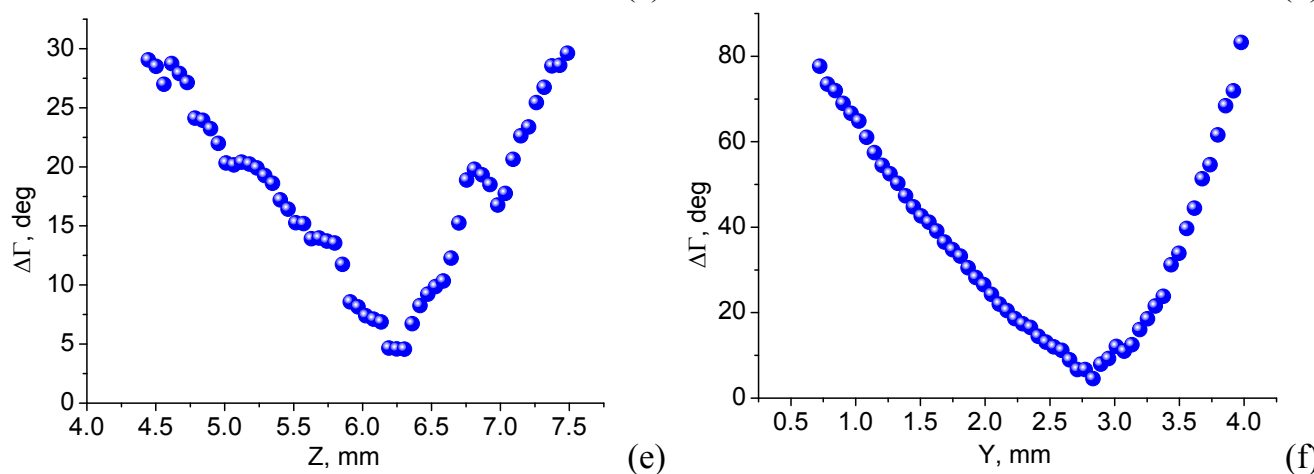

Fig. 5. Dependences of the phase difference at $\sigma_{3}^{\prime}=0$ in the vicinities of defect cores in $\mathrm{CaB}_{4} \mathrm{O}_{7}$ glass, as obtained for $\operatorname{TD}_{1}(a, b), \mathrm{TD}_{2}(\mathrm{c}, \mathrm{d})$ and $\mathrm{TD}_{2}(\mathrm{e}, \mathrm{f})$ under the condition $\sigma_{3}^{\prime}=-13.48 \times 10^{6} \mathrm{~N} / \mathrm{m}^{2}$.

For example, in the simplified situation when we have $k_{4}=k_{1}$ and $k_{5}=k_{2}$, Eq. (10) reduces to $\tan 2 \zeta_{1}=-\tan \left(\varphi+\varphi_{0}\right) \quad$ or $\quad \zeta_{1}=-\left(\varphi+\varphi_{0}\right) / 2, \quad$ where $\quad \varphi_{0}=\arctan \left(k_{1} / k_{2}\right), \quad Y=\rho \cos \varphi$, $Z=\rho \sin \varphi$, and $(\rho, \varphi)$ are the polar coordinates. Moreover, the TDs would appear even when $k_{1} \neq 0$ or $k_{2} \neq 0$. It can happen whenever $\left(\operatorname{Rot} P_{3}\right)_{1}=k_{1} \neq 0$ and $\left(\operatorname{Rot} P_{2}\right)_{1}=-k_{4} \neq 0$ or 


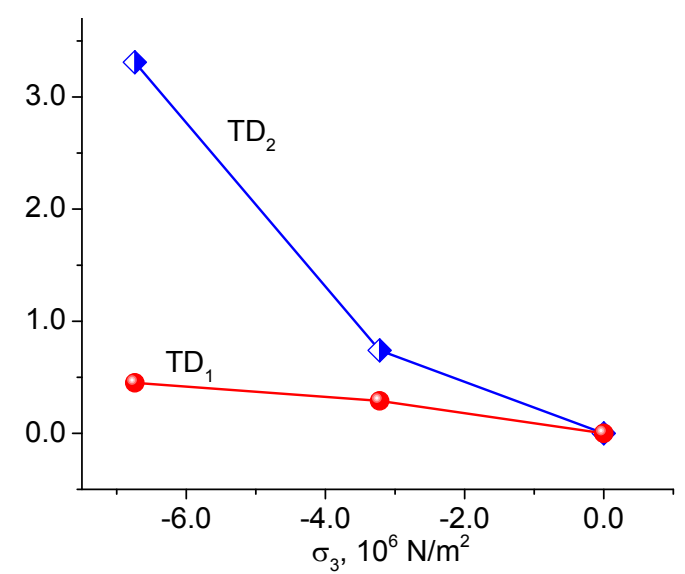

Fig. 6. Dependence of displacement of the TDs on the compressive stress, as obtained for $\mathrm{CaB}_{4} \mathrm{O}_{7}$ glass sample.

$\left(\operatorname{Rot} P_{2}\right)_{1}=k_{2} \neq 0$ and $\left(\operatorname{Rot} P_{3}\right)_{1}=k_{5} \neq 0$, where $P_{3}$ and $P_{2}$ are the load that are determined by the applied force $F_{\mathrm{i}}$ per unit area $S$, i.e. $P_{\mathrm{i}}=F_{\mathrm{i}} / S$. Notice also that, under torsion of $\mathrm{LiNbO}_{3}$ crystals around the $Z$ axis, the relations $\left(\operatorname{Rot} P_{1}\right)_{3} \neq 0$ and $\left(\operatorname{Rot} P_{2}\right)_{3} \neq 0$ should be true for inducing the TDs $[14,15]$. On the other hand, under bending of a glass beam by a moment $M_{3}$, the relations $\left(\operatorname{Rot} P_{1}\right)_{3} \neq 0$ and $\left(\operatorname{Rot} P_{2}\right)_{3} \neq 0$ should be satisfied for the same aim [12]. Hence, we are in a position to formulate the general conditions needed for the appearance in the glasses of the TDs with the strength $\pm 1 / 2$ : (i) a $2 \mathrm{D}$ stress distribution in the vicinity of the defect core, (ii) the condition $\left(\operatorname{Rot} P_{k}\right)_{l} \neq 0$, and (iii) a linear dependences of the stress components on the coordinates holding true around the defect core.

Now let us analyze the behaviour of our TDs under condition when the homogeneous compressive stress $\sigma_{3}^{\prime}$ is applied. Then Eq. (6) may be rewritten as

$$
\begin{aligned}
& \left(B_{1}+\pi_{11} \sigma_{2}+\pi_{12}\left(\sigma_{3}+\sigma_{3}^{\prime}\right)\right) Y^{2}+\left(B_{1}+\pi_{12} \sigma_{2}+\pi_{11}\left(\sigma_{3}+\sigma_{3}^{\prime}\right)\right) Z^{2}+ \\
& 2\left(\pi_{11}-\pi_{12}\right) \sigma_{4} Y Z=1
\end{aligned}
$$

while Eqs.(7) and (8) become as follows:

$$
\begin{aligned}
& \Delta n_{23}=-\frac{n^{3}}{2}\left(\pi_{11}-\pi_{12}\right) \sqrt{\left(\left(k_{4}+k_{1}\right) Z-\left(k_{2}+k_{5}\right) Y-\sigma_{3}^{\prime}\right)^{2}+4\left(k_{1} Y+k_{2} Z\right)^{2}}, \\
& \tan 2 \zeta_{1}=\frac{2\left(k_{1} Y+k_{2} Z\right)}{\left(k_{4}+k_{1}\right) Z-\left(k_{2}+k_{5}\right) Y-\sigma_{3}^{\prime}} .
\end{aligned}
$$

We have simulated the spatial map of the angle of OI rotation for the defect $\mathrm{TD}_{2}$, using Eq. (14) and the average parameters $k_{1}=-19.8 \times 10^{9}, \quad k_{2}=-1.98 \times 10^{9}, \quad k_{4}=3 \times 10^{9}$ and $k_{5}=9 \times 10^{9} \mathrm{~N} / \mathrm{m}^{3}$, and $\sigma_{3}^{\prime}=-3.22 \times 10^{6} \mathrm{~N} / \mathrm{m}^{2}$. It is seen from Fig. 7 that the application of the compressive mechanical stress can displace the TD of OI orientation approximately by $0.2 \mathrm{~mm}$.

Finally, the coordinates of the TD can be obtained by solving the system of equations based on the uncertainty of the angle of OI rotation:

$$
\left\{\begin{array}{l}
k_{1} Y+k_{2} Z=0 \\
\left(k_{4}+k_{1}\right) Z-\left(k_{2}+k_{5}\right) Y-\sigma_{3}^{\prime}=0
\end{array} .\right.
$$



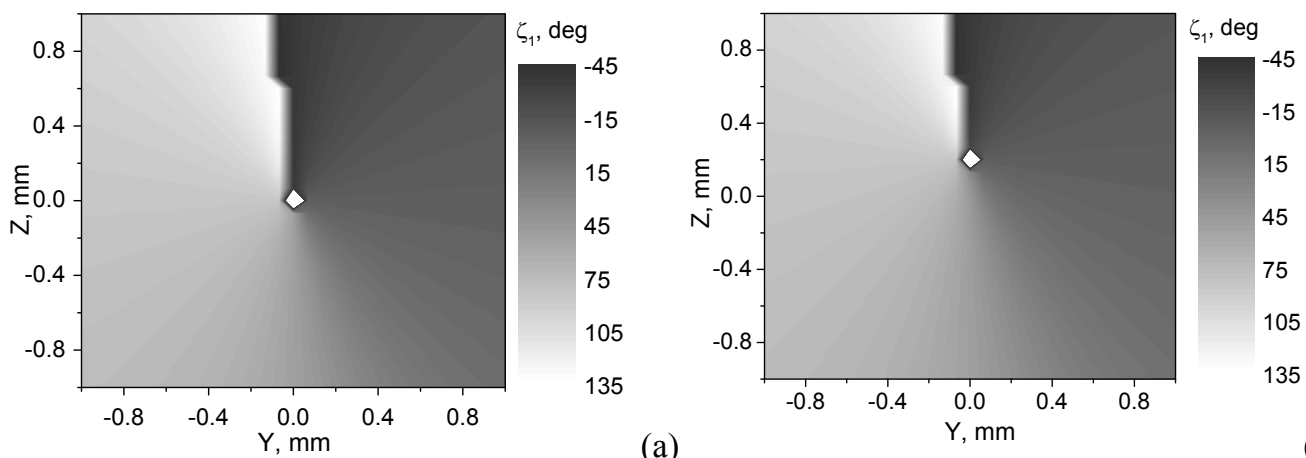

(a)

Fig. 7. TDs of OI orientation appearing at $\sigma_{3}^{\prime}=0$ (a) and $\sigma_{3}^{\prime}=-3.22 \times 10^{6} \mathrm{~N} / \mathrm{m}^{2}(\mathrm{~b})$.

For the parameter values mentioned above, we get the following coordinates: $(Y, Z)=(-0.01 \mathrm{~mm}$, $0.2 \mathrm{~mm}$ ). It is obvious that, with some values of the $k_{i}$ parameters and the external mechanical stresses, one can reach the condition under which the TDs leave out of the sample. Moreover, it is quite possible that the topological reaction of adding of the defects $\left(\mathrm{TD}_{1}+\mathrm{TD}_{2}=1 / 2+1 / 2=1\right)$ can be realized while one chooses properly the parameters mentioned above.

\section{Conclusions}

In the present work we have experimentally studied the movement of the TDs of OI orientation, which are caused by the residual stresses available in the $\mathrm{CaB}_{4} \mathrm{O}_{7}$ glass, under application of external compressive mechanical stress. We have found that the TDs change their positions inside the sample and the dependence of this displacement on the uniaxial mechanical stresses is close to linear. The observed effect is explained basing on the equation of OI perturbed by the residual and external mechanical stresses. We have ascertained that the TDs of OI orientation are nothing but the linear defects which represent some chains inside the sample surrounded by the regions of 2D spatial distributions of the phase difference and, hence, of the residual stresses.

As a result of our experimental and phenomenological results, we have formulated the following conditions needed in order that the TDs of OI orientation with the strength module $|1 / 2|$ appear in the glasses: (i) a 2D stress distribution should take place in the vicinity of the defect core, (ii) the conditions $\left(\operatorname{Rot} P_{k}\right)_{l} \neq 0$ should hold true, and (iii) the stress components should depend linearly on the coordinates around the defect core.

Following from the present analysis, one can assume that, in principle, the TDs can leave the glass sample under some conditions defined by the spatial distribution of the residual stress tensor components and the values of the external stresses applied to the sample. Moreover, it is quite probable that one can observe the appropriate topological reactions when the parameters mentioned above are chosen properly.

\section{References}

1. Zurek W H, 1985. Cosmological experiments in superfluid helium? Nature. 317: 505-508.

2. Dirac P, 1931. Quantised singularities in the electromagnetic field. Proc. Roy. Soc. (London) A. 133: $60-72$.

3. Georgi H and Glashow S L, 1974. Unity of all elementary particle forces. Phys. Rev. Lett. 32: 438-441.

4. Zheludev I S. Symmetry and its application. Moscow: Atomizdat (1976).

5. Trebin H.-R., 1998. Defects in liquid crystals and cosmology. Liq. Cryst. 24: 127-130. 
6. Kurik M V and Lavrentovich O D, 1988. Defects in liquid crystals: homotopy theory and experimental studies, Sov. Phys. Uspekhi. 31: 196-224.

7. Vasylkiv Yu., Skab I. and Vlokh R., 2014. Generation of double-charged optical vortices on the basis of electro-optic Kerr effect. Appl. Opt. 53: B60-B73.

8. Skab I, Vasylkiv Yu, Smaga I and Vlokh R, 2011. Spin-to-orbital momentum conversion via electro-optic Pockels effect in crystals. Phys. Rev. A. 84: 043815.

9. Vasylkiv Yu, Skab I and Vlokh R, 2014. Crossover regime of optical vortices generation via electrooptic nonlinearity: the problem of optical vortices with the fractional charge generated by crystals. J. Opt. Soc. Amer. A. 31: 1936-1945.

10. Vasylkiv Yu., Kryvyy T., Skab I. and Vlokh R. 2014. Behavior of topological defects of optical indicatrix orientation in cubic single crystals under conically distributed electric field. 1. The electric field and the optical beam parallel to the three-fold symmetry axis. Ukr. J. Phys. Opt. 15: 184-194.

11. Vasylkiv Yu., Kryvyy T., Skab I. and Vlokh R. 2015. Behaviour of topological defects of optical indicatrix orientation in cubic, hexagonal, trigonal and tetragonal crystals under conically distributed electric field. 2. The electric field and the optical beam parallel to the principal crystallographic directions. Ukr. J. Phys. Opt. 16: 1-16.

12. Skab I, Vasylkiv Yu and Vlokh R, 2012. Induction of optical vortex in the crystals subjected to bending stresses. Appl. Opt. 51: 5797-5805.

13. Skab I, Vasylkiv Yu, Krupych O, Savaryn V and Vlokh R, 2012. Generation of doubly charged vortex beam by concentrated loading of glass disks along their diameter. Appl. Opt. 51: $1631-1637$.

14. Skab I, Vasylkiv Yu, Savaryn V and Vlokh R, 2011. Optical anisotropy induced by torsion stresses in $\mathrm{LiNbO}_{3}$ crystals: appearance of an optical vortex. J. Opt. Soc. Amer. A. 28: 633640.

15. Skab I, Vasylkiv Yu, Zapeka B, Savaryn V and Vlokh R, 2011. Appearance of singularities of optical fields under torsion of crystals containing threefold symmetry axes. J. Opt. Soc. Amer. A. 28: 1331-1340.

16. Vasylkiv Y, Skab I, Smyk M and Vlokh R, 2014. Topological defects of optical indicatrix orientation in stressed glasses: spatial distribution of optical anisotropy parameters. Appl. Opt. 53: 3967-3975.

17. Vasylkiv Yu, Skab I and Vlokh R, 2016. Identification of the topological defects of optical indicatrix orientation in $\mathrm{CaB}_{4} \mathrm{O}_{7}$ glasses. J. Opt. (to be published).

18. Adamiv V T, Burak Ya V, Gamernyk R V, Romanyuk M M and Teslyuk I M, 2011. Optical properties of alkali and alkaline earth tetraborate glasses prepared in the alumina crucible. Functional Mater. 18: 298-303.

Vasylkiv Yu., Kryvyy T., Skab I. and Vlokh R. 2016. Conditions of appearance of the topological defects of optical indicatrix orientation in the glasses with residual stresses: Movement of the defects under application of external mechanical stress to $\mathrm{CaB}_{4} \mathrm{O}_{7}$ glasses. Ukr.J.Phys.Opt. 17: $65-74$

Анотація. У роботі експериментально виявлено зміщення топологічних дефектів орієнтації оптичної індикатриси, пов'язаних з існуванням залишкових напружень у склі $\mathrm{CaB}_{4} \mathrm{O}_{7}$, під дією стискаючого механічного напруження. Спостережуваний ефект пояснено на основі рівняння оптичної індикатриси, збуреної залишковими напруженнями $i$ прикладеним механічним напруженням. Сформульовано умови появи в склі топологічних дефектів орієнтації оптичної індикатриси з модулем сили $|1 / 2|$ і обговорено можливість топологічних реакцій, спричинених прикладеним напруженням. 